\title{
Frames als Repräsentationsformat in modernen Terminologiesystemen
}

\author{
Birte Lönneker-Rodman \& Alexander Ziem
}

\begin{abstract}
Der vorliegende Beitrag stellt ein konkretes Anwendungsgebiet von semantischen Frames im Bereich der praktischen Terminologiearbeit, einem Untergebiet der Lexikografie, vor. Es wird insbesondere gezeigt, wie Frames mittels existierender Software zur Terminologieverwaltung dargestellt werden können und welcher konkrete praktische Nutzen sich daraus ergibt. Nach einer kurzen Einführung in das Anwendungsgebiet der Terminologieforschung und einschlägige Software wird dargelegt, inwiefern sich konzeptuelle Informationen, die mittels Frames erfasst werden, für die Terminologiearbeit verwenden lassen. Ausgeführt wird, wie Frame-Ansätze auf den Bereich der Terminologiearbeit übertragen werden können und welche Möglichkeiten es gibt, Frames mit marktüblicher Software zu modellieren. Ein besonderes Augenmerk liegt hier auf dem Nutzen und den Beschränkungen aktueller Software-Tools. Abschließend werden die erzielten Ergebnisse zusammengefasst und im Hinblick auf mögliche zukünftige Anwendungsfelder bewertet.
\end{abstract}

\section{Einleitung}

Frames spielen nicht nur in bedeutungstheoretischen Grundlagendiskussionen eine zunehmend große Rolle (Busse 2012; Löbner 2014; Ziem 2014a), auch ihr praktischer Einsatz in so unterschiedlichen Anwendungsbereichen wie der Psychiatrie (hier etwa zur Erstellung von Klassifikationssystemen, vgl. Vosgerau/ Zielasek/Soom in diesem Band), der Übersetzungswissenschaft (López 2002; Čulo 2013), der computerbasierten Sprachverarbeitung (Das et al. 2013) und der Terminologiearbeit in Fachdomänen (Faber/López Rodríguez 2012; Faber 2015) ist in der letzten Dekade vielseitiger und umfassender geworden. Das Frame-Konzept 
hat dabei im Zuge linguistischer, philosophischer, medien-, kognitions- und computerwissenschaftlicher Modellierungen von Wissen in zweifacher Form Eingang gefunden. Zum einen dienen Frames als Analyseinstrumente, um Wissen strukturell zu beschreiben und analytisch fassbar zu machen; dies ist etwa der Fall, wenn sie als Annotationsschemata (etwa Fraas/Pentzold/Ziem in diesem Band) oder als Werkzeug für qualitative Inhaltsanalysen (Scheufele in diesem Band) eingesetzt werden. Zum anderen gelten Frames als ein Format, in dem Wissen kognitiv repräsentiert ist; dieser Anspruch ist bereits in Fillmores linguistischer Frame-Konzeption angelegt, in aller Deutlichkeit wird er von dem kognitiven Psychologen Barsalou (1992) vertreten und im Düsseldorfer Frame-Ansatz fortgesetzt. In dem vorliegenden Beitrag verstehen wir Frames in diesem doppelten Sinne zugleich als ein analytisches Hilfsmittel und ein kognitives Repräsentationsformat, möchten insbesondere aber, anders als die meisten Beiträge in diesem Band, den praktischen Nutzen von Frames herausstellen. Konkret geschieht dies am Beispiel von Wissensdarstellungen und -erschließungen durch Frames in modernen Terminologiesystemen.

Die linguistische Beschäftigung mit Terminologie und Terminologiesystemen kann, vereinfacht gesagt, als ein Untergebiet der Lexikografie aufgefasst werden. Aufgrund der besonderen Bedeutung der Terminologiearbeit wie auch der Lexikografie für die Praxis und für die in diesen Bereichen verwendeten SoftwareSysteme lohnt es sich, den Gegenstandsbereich präziser zu konturieren. Sager $(1990,3)$ versucht, Terminologie wie folgt von Lexikografie abzugrenzen:

By its etymology 'terminology' would mean 'the science/study/knowledge of terms' which would make it parallel to lexicology, the science/study/knowledge of the lexicon or lexical items; this interpretation is, however, rejected by most terminologists. [...] ['Terminology' is used] to refer to an internally consistent and coherent set of terms belonging to a single subject field [...]. (Hervorhebung hinzugefügt)

Auch Faber und López Rodrígez $(2012,12)$ heben die Bedeutung des Fachgebiets für die Terminologie hervor. Sie verstehen unter Terminologie den Bestand von Einheiten (,units“) eines Fachgebiets. Hiermit ist noch nicht gesagt, ob dieser Bestand (vorrangig) von der sprachlichen oder der konzeptuellen Ebene her erfasst und strukturiert ist.

In der Praxis besteht eine gängige Auffassung in der Annahme, dass es sich bei einer Terminologiesammlung um ein „Wörterbuch“ handele, dessen Inhalte sich 
auf ein bestimmtes Fachgebiet beziehen, beispielsweise auf eine Branche, ein Unternehmen oder eine Abteilung. Damit ist das Fachgebiet einerseits das vereinende Element, also der Lebens-, Arbeits- und Gedankenraum, innerhalb dessen die in einer bestimmten Terminologie betrachteten Dinge, Konzepte oder sprachlichen Zeichen eine Rolle spielen. Andererseits wird eine Abgrenzung zu anderen Fachgebieten vorgenommen, in denen die betrachteten Einheiten ebenfalls eine Rolle spielen bzw. spielen können. Sofern die anderen Fachgebiete für die betreffende Branche oder das Unternehmen nicht ebenfalls relevant sind, können derartige Mehrdeutigkeiten also bei der Terminologiepflege ignoriert werden.

Aufgrund der Konzentration auf sehr spezifische Fachgebiete sind oft neben den Erstellern von Terminologiesammlungen oder Terminologiedatenbanken, d. h. neben den Terminologen und ihren Informanten, auch die Anwender bzw. Nutzer von Terminologieverwaltungssystemen hochgradig spezialisiert. Je nach Arbeitsgebiet handelt es sich bei letzteren z. B. um Elektrotechniker, Marketingexperten oder Mitarbeiter in Support und Wartung technischer Produkte.

Das Ziel des vorliegenden Beitrages besteht darin aufzuzeigen, inwiefern Frames durch existierende Software zur Terminologieverwaltung so verwendet werden können, dass sich bestehende Defizite bei der professionellen Verwaltung von Fachtermini vermeiden lassen. In einem ersten Schritt umreißen wir das Anwendungsgebiet der Terminologieforschung und der dafür einschlägigen Software, wobei ein besonderes Augenmerk auf der Relevanz von konzeptuellen Informationen für die Terminologiearbeit liegt (Abschnitt 2). Im Anschluss daran stellen wir Möglichkeiten vor, Frames in Terminologiesystemen als Darstellungsformat zu integrieren. Am Beispiel von Verpackungsmaschinen machen wir dabei von einem hybriden Frame-Modell Gebrauch, das sowohl Aspekte von Minskys Frame-Konzeption aufgreift als auch linguistische Weiterentwicklungen im Rahmen des Berkeleyer FrameNet-Projektes berücksichtigt (Abschnitt 3). Von besonderem Nutzen erweisen sich Möglichkeiten zur Modellierung und grafischen Darstellung von terminologischen Netzwerken; ihnen wenden wir uns abschließend zu (Abschnitt 4). 


\section{Terminologie als praktische Anwendungsdomäne: Hintergrund und Herausforderungen}

\subsection{Von Wortlisten zum Terminologiemanagementsystem}

Die einfachsten Terminologiesammlungen sind Wortlisten, in der Terminologielehre häufig als „Glossare“ bezeichnet. Diese Listen sollen je nach Beschaffenheit entweder als Übersetzungs- oder als Formulierungshilfe dienen. Als Übersetzungshilfe fungieren in der Regel zwei- oder mehrsprachige Wortlisten, wie in Tabelle 1 beispielhaft illustriert; in die Kategorie der Formulierungshilfe fallen dagegen einsprachige Wortlisten, die nach ihrer empfohlenen Verwendung strukturiert sind.

\begin{tabular}{ll}
\hline Englisch & Deutsch \\
\hline garden & Garten \\
\hline weed & jäten \\
\hline weed & Unkraut \\
\hline water & Wasser \\
\hline water & begießen \\
\hline bank & Böschung \\
\hline excavator & Bagger \\
\hline$\ldots$ & $\ldots .$. \\
\hline
\end{tabular}

Tabelle 1: Zweisprachiges Glossar

Hintergrund einer Kennzeichnung von „empfohlenen“ vs. „nicht zu verwendenden" Ausdrücken ist das Bestreben, den Sprachgebrauch der Terminologie-Konsumenten aus welchen Gründen auch immer zu normieren (mehr dazu in den Abschnitten 2.2 und 2.3). Ein Beispiel hierfür ist in Tabelle 2 illustriert.

Der Bestand dieser Glossare ist mithin vorrangig von der sprachlichen Ebene her erfasst und strukturiert - abgesehen vom Zusammenhang über das betrachtete Fachgebiet hilft die Liste dem Empfänger nicht, die Bedeutung der enthaltenen Wörter und ihre Beziehungen untereinander zu erfassen. Glossare haben 
aber den Vorteil, dass sie verhältnismäßig schnell erstellt werden können. Für den Autor eines Glossars, der dieses in nicht zu großem zeitlichem Abstand selbst als Konsument zur Hand nimmt, kann die einfache Struktur sogar ein Vorteil sein. Man denke in diesem Zusammenhang etwa an eine Vokabelliste als Lernhilfe oder einen Spickzettel (im Bereich Terminologie für Dolmetscher vgl. auch Rütten 2013).

\begin{tabular}{ll}
\hline Empfohlen & Nicht verwenden \\
\hline Wildkraut & Unkraut \\
\hline Herbizid & Unkrautvernichtungsmittel \\
\hline bewässern & begießen \\
\hline$\ldots$ & $\ldots$. \\
\hline
\end{tabular}

Tabelle 2: Einsprachiges Glossar mit Verwendungshinweisen

Die Nachteile der Wortliste kommen deutlich zum Vorschein, wenn die Liste über längere Zeiträume und/oder von unterschiedlichen Personen verwendet werden soll. Zum einen kann ein Glossar nicht zwischen mehreren Bedeutungen eines Wortes unterscheiden (z. B. water - water, bank - bank). Selbst bei der Begrenzung auf ein einziges Fachgebiet ist es möglich, dass eine solche Mehrdeutigkeit vorkommt. Ein Glossar bietet in diesem Fall keine Hilfe zur Disambiguierung, also keine Entscheidungshilfe dafür, welche fremdsprachliche Entsprechung (von zwei oder mehr möglichen) bzw. welchen bevorzugten Term (von mehreren möglichen) der Anwender zu benutzen hat. Zum anderen gibt das Glossar keinen Hinweis darauf, ob dem Anwender beim Nachschlagen überhaupt die korrekte Bezeichnung eingefallen ist. Wenn der Anwender beispielsweise irrtümlich eine Mutter als "Schraube“ bezeichnet und Schraube nachschlägt, wird er den Term Schraube übersetzen bzw. prüfen. Solange das Glossar keinerlei konzeptuellen Informationen enthält, kann es dem Anwender also nicht helfen, falls sein Wortgebrauch auf ein ,falsches', d. h. im jeweiligen Verwendungskontext nicht-intendiertes Ding in der Welt verweist.

Natürlich ließen sich Wortlisten-Darstellungen wie in Tabelle 1 und 2 um zusätzliche Spalten erweitern und ggf. sogar zusammenführen, sodass sie weiteren zur Disambiguierung benötigten Informationen Platz bieten. Zum Beispiel 
könnte eine Spalte „Wortart“ eingefügt werden, über die sich die englischen Terme water (Verb) und water (Substantiv) unterscheiden ließen. Dadurch wird die Tabelle aber schnell unübersichtlich und unhandlich. Die Anwender bzw. Nutzer verbrauchen für das Nachschlagen und die Orientierung in der angereicherten Tabelle viel Zeit - oder sie unterlassen das Nachschlagen gänzlich, um Zeit zu sparen, wodurch aber der beabsichtigte Nutzen der Terminologiearbeit nicht erzielt wird.

Um diesen Problemen Rechnung zu tragen, werden seit geraumer Zeit computerbasierte Terminologiesysteme - auch bekannt als „Terminologiemanagementsystem“, „Terminologieverwaltungssystem“, „Terminologiedatenbank“ - entwickelt. Viele dieser Systeme gehen über die Anlage von Wortlisten weit hinaus, insofern in den Systemen etwa Terme unterschiedlicher Sprachen angelegt und mit zusätzlichen Informationen, die der Disambiguierung von Termen dienen, gespeichert werden können. Auf diese Weise wird nicht nur dem Nutzer die Suche im Terminologiebestand erleichtert; die ermittelten Informationen werden darüber hinaus auch übersichtlich angezeigt.

Terminologiesysteme sind entweder alleinstehende Softwareprodukte oder stehen als Module zur Verfügung, die in andere Systeme eingebettet sind. Als Module sind sie typischerweise Bestandteil von Übersetzungsmanagementsystemen und Computer Aided Translation bzw. von Content Management Systemen oder auch von Software zur Autorenunterstützung. Die Einbettung in andere Systeme oder die Verknüpfung mit anderen Anwendungen ist für den Nutzer insofern besonders hilfreich und zeitsparend, als das Nachschlagen von Fachwörtern in einem bestimmten Anwendungsprogramm und auch innerhalb eines bestimmten sprachlichen Kontextes erfolgt. Das System kann also die Terme während des Übersetzens oder Schreibens bereits selbst suchen und die Ergebnisse im Terminologie-Modul anzeigen. Der Anwender braucht in seiner Tätigkeit nicht innezuhalten, um zu überlegen, ob passende fachspezifische Terme vorliegen, die in einer separaten Anwendung gesucht werden müssten.

\subsection{Ziele der Terminologiearbeit}

Während die Kosten der Terminologiearbeit relativ leicht zu beziffern sind, besteht der Nutzen in verschiedenen Faktoren, die sich teilweise nur schwer bzw. indirekt messen lassen. Hierzu zählen: 
- Einsparungen (vor allem an Arbeitszeit, ggf. auch an Material wie Fehldrucken);

- Verringerung von Risiken (z. B. Regressansprüche aufgrund unverständlicher oder falscher Bedienungsanleitungen, Beipackzettel usw.);

- Erhöhung von Qualität (Produktqualität, Arbeitsumfeld);

- Verbesserung des Images der die Terminologie verwendenden Organisation.

Auf einer allgemeinen Ebene verfolgt eine Institution mit dem Aufbau und der Pflege einer Terminologiedatenbank also zwei Ziele, nämlich Eindeutigkeit und Verständlichkeit: ${ }^{1}$

Eindeutigkeit. Je eindeutiger Texte sind, desto weniger Missverständnisse entstehen. Zum Beispiel sollten die Marketing-Abteilung (Katalog, Online-Shop), die Forschungs- und Entwicklungsabteilung sowie die Technische Support-Abteilung für das gleiche Bauteil eines Produkts dieselbe Benennung verwenden. Ansonsten bestünde die Gefahr, dass ein Kunde ein vom Support empfohlenes Ersatzteil im Katalog nicht findet bzw. ein falsches Ersatzteil identifiziert. Beim Vorliegen mehrerer möglicher Bezeichnungen (z. B. Antriebswellenmanschette, Achsmanschette, Gelenkschutzhülle) sollte also eine sogenannte Vorzugsbenennung bestimmt (z. B. Antriebswellenmanschette) und andere Bezeichnungen nur unter bestimmten Bedingungen erlaubt (z. B. Achsmanschette) oder gänzlich vermieden werden (z. B. Gelenkschutzhülle). Ähnlich ist es mit der Benennung von Tarifen (z. B. Telefontarifen, Stromtarifen mit Benennungen wie Light, Paket L, Large, Package L usw.), die bei Bestellungen, Abrechnungen und im Werbematerial eindeutig identifizierbar sein müssen, damit nicht z.B. Light bestellt und Large abgerechnet wird. Die Terminologiedatenbank gibt bei mehreren denkbaren Bezeichnungen darüber Aufschluss, welche davon (per Konvention) verwendet werden soll.

Verständlichkeit. Je verständlicher die gesprochenen und geschriebenen Texte sind, die ein Unternehmen intern und extern verwendet bzw. veröffentlicht, desto besser erschließen sie sich. Die Verständlichkeit, aber auch die Glaubwürdigkeit der Fachtexte werden zu einem großen Teil durch den verwendeten Fachwortschatz bedingt (vgl. Faber/San Martín 2012, 196 f.). In Fachtexten für Laien bzw.

1 Auch der Deutsche Terminologie-Tag e. V. (2014: M8-3) nennt diese beiden Aspekte als den Nutzen von Terminologie, nämlich zum einen die "gemeinsame Sprache aller Beteiligten von Anfang an“ und zum anderen die „Brücke zum Sprachgebrauch anderer“. 
„nicht Eingeweihte“, z. B. Interessenten, Kunden oder Geschäftspartner, müssen also Terme für unternehmens- oder produktspezifische Besonderheiten des Anbieters zunächst erklärt werden. Um die Glaubwürdigkeit zu sichern, sollten Terme des Fachgebiets gerade nicht vermieden werden, sondern korrekt und konsistent eingesetzt werden. Bis zu einem gewissen Grad müssen gesprochene und geschriebene Texte die Adressaten auch in die Fach-Materie einführen, z. B. in Spezifika bei Unternehmensabläufen, Produkten usw. Hier gilt es, auch neue Mitarbeiter möglichst schnell mit dem Fachwortschatz vertraut zu machen.

\subsection{Präskriptive vs. deskriptive Terminologiearbeit}

Im wissenschaftlichen Kontext wirkt der normierende bzw. präskriptive Charakter der Terminologiearbeit zur Erreichung von Eindeutigkeit zunächst befremdlich. Der Zweck der Eindeutigkeit geht auf eine Annahme von Eugen Wüster zurück (siehe dazu Faber/López Rodríguez 2012, 12 ff.), derzufolge per Normierung oder Standardisierung der (Fach-)Sprache eine 1:1-Beziehung zwischen Konzepten und ihren Benennungen hergestellt werden kann. Sowohl Synonyme als auch Homografen (Homonyme) sollen seiner Auffassung nach also vermieden werden.

Synonyme treten dann auf, wenn ein Konzept mehrere Benennungen hat, z. B. Schnürband - Schnürsenkel - Schuhband oder Aufzug - Fahrstuhl - Lift. Nach Wüster und anderen Vertretern seiner "General Terminology Theory“ wäre es angeraten, einen Ausdruck als Vorzugsbenennung zu definieren und die anderen beiden zu verbieten, da bei bestimmten Textsorten eine geringere Varianz Vorteile bringt. Sie kann etwa dazu beitragen, dass eine Bedienungsanleitung klarer wird. Der Leser muss nicht überlegen, ob mit Schuhband auf dasselbe Konzept Bezug genommen wird wie etwa mit Schnürband, da nur Schnürband, nicht aber Schuhband verwendet wird. Beim Übersetzen von Texten ergibt sich ein Einsparungseffekt, wenn im Ausgangstext Synonyme nicht wahlweise abwechselnd verwendet werden, sondern lediglich die Vorzugsbenennung. Ein Übersetzer muss nicht mehrere Synonyme nachschlagen und wortwörtliche Satzwiederholungen sind kostengünstiger als neue bzw. ähnliche Sätze, die durch die Verwendung eines Synonyms entstehen.

Eine andere Herausforderung stellen Homonyme dar, wie etwa Maus (Fachbereich Biologie) und Maus (Fachbereich IT) oder Bank (als eine Finanzinstitution) 
und Bank (als ein Sitzmöbel). ${ }^{2}$ Ziel wäre es, eine Verwechslung zu vermeiden, indem eine dieser Benennungen verboten und durch eine andere, ein Synonym, ersetzt wird. Beispielweise könnte ein Terminologe statt Maus die genauere Benennung Computermaus und für Bank die genauere Bezeichnung Sitzbank vorschreiben. ${ }^{3}$

Trotz der genannten Vorteile birgt die präskriptive Terminologie auch Nachteile. Bei der Erstellung lässt sich nicht unbedingt vorhersehen, welche Homonyme zu Verwechslungen führen könnten. Wenn zur Vermeidung von möglichen Verwechslungen die Verwendung präziser Benennungen wie Computermaus und Flachkopfschraube überall gefordert wird, führt dies zu schwerfälligen Texten. Somit wäre die gewünschte Verständlichkeit sogar wieder gemindert.
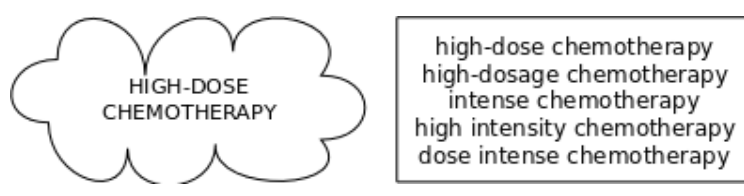

intensive chemotherapy aggressive chemotherapy dose intensive chemotherapy intensified chemotherapy etc.

Abb. 1: Konzept mit mehreren Benennungen (nach Faber/San Martín 2012, 190)

Warum es gerade in der Fachsprache so viele Synonyme (sowohl Benennungsvarianten als auch formale Varianten) gibt, ist eine in der sprachwissenschaftlichen Forschung bisher unterrepräsentierte Fragestellung (vgl. Faber/San Martín 2012, 189 ff.). Faber und San Martín $(2012,190)$ haben eine Reihe von Benennungen für das Konzept HIGH-DOSE CHEMOTHERAPY zusammengetragen (vgl. Abb. 1). Ohne weitere Erläuterungen wird sich ein Autor eines medizinischen oder tech-

2 Im Falle von Bank führt Metonymie zu weiteren, hier nicht weiter betrachteten, Homonymen, wie z. B. Gebäude oder Vertreter der Finanzinstitution.

3 Dies ist nur bedingt sinnvoll, da gerade aufgrund der unterschiedlichen Fachgebiete die Benennungen selten im gleichen Text auftreten. Solche Texte müssten Anteile an beiden Fachgebiete haben, z. B. eine Benutzungsanleitung für ein zoologisches Softwareprodukt oder ein innenarchitektonisches Konzept für ein Bankgebäude. Der Terminologe hat mit der Entscheidung, ob die Unterscheidung zwischen Bank und Bank relevant ist, mindestens eine Festlegung auf der kognitiven Ebene bereits getroffen. Er hat entschieden, welches Fachgebiet oder welche Fachgebiete seine Terminologiesammlung abdeckt. Wenn die Terminologiedatenbank nur das Fachgebiet der Innenarchitektur abdeckt, ist die Erfassung der Bank als Sitzmöbel ausreichend. Unter Anwendung der sogenannten „Closed World Assumption“ kann die Beschreibung der Bank als Finanzinstitut aus der Innenarchitektur-Terminologiesammlung unbeachtet bleiben. 
nischen Fachtextes nicht von einem Terminologen vorschreiben lassen, nur bestimmte Ausdrücke zu benutzen bzw. die Verwendung von (teil-)synonymen Ausdrücken gänzlich zu vermeiden.

Eine Terminologiesammlung kann daher auch nach dem deskriptiven Prinzip erstellt werden, indem man die (in der Terminologielehre weit verbreitete) Forderung einer Vorzugsbenennung weglässt. Beim Erstellen einer deskriptiven Ressource geht es sowohl um die Erfassung von Benennungen als auch um die Erfassung bzw. Dokumentation von Wissen über die dargestellten sprachlichen sowie konzeptuellen Einheiten des Fachgebiets. Bei der Rezeption wird dieses Wissen durch den einzelnen Empfänger erschlossen. Deskriptive Terminologie-Ressourcen dienen ebenfalls der Erhöhung der Verständlichkeit von Texten, vor allem durch die Ermöglichung der angemessenen Verwendung des Fachwortschatzes beim Schreiben. Darüber hinaus besteht ein weiteres Einsatzgebiet deskriptiver Terminologie-Ressourcen darin, dem Rezipienten als Nachschlagewerke oder als Verständnishilfe beim Lesen von Texten oder bei der Aufbereitung anderer Kommunikationen zu dienen. Terminologie-Ressourcen können auch explorativ verwenden werden, zum Beispiel beim Einarbeiten in ein neues Fachgebiet, sei es als Übersetzer oder als neuer Mitarbeiter eines Unternehmens. Schließlich können neue Produkte oder neue Funktionen und Technologien innerhalb der Terminologiedatenbank zunächst beschrieben werden. Bei Bedarf ließe sich sogar der Prozess ihrer Namensfindung im Terminologie-System abbilden.

Deskriptive Terminologiearbeit nützt auch dem Übersetzer. Zwar wird eine Fachterminologie-Datenbank häufig mehrsprachig gepflegt. Falls dem Übersetzer aber die verwendeten Fachterme oder die benannten Dinge oder Prozesse des Fachgebiets nicht bekannt sind, kann das zu einer geringeren Verständlichkeit des gesamten ausgangssprachlichen Satzes führen und somit die Übersetzung erschweren. Hilfreich ist also, wenn das Konzept ausführlicher beschrieben ist, sodass der Übersetzer das Domänenwissen direkt in der Terminologie-Ressource erwerben kann. Außerdem sollen gerade Übersetzer auch die „Übersetzungen” für zunächst einsprachig existierende Terminologiebestände erstellen. Hierzu lesen sie einen einsprachigen Terminologie-Eintrag und tragen dann die fremdsprachliche Entsprechung als neuen Term ein. Ohne weitere erläuternde Informationen auf Konzept- und Termebene ist eine solche „Term-Übersetzung“ eine große Herausforderung, weil - abgesehen vom Fachgebiet - der Kontext fehlt. 


\subsection{Terminologie und Wissensrepräsentation}

Insbesondere im Fall der deskriptiven, aber auch der präskriptiven Terminologiearbeit sind Theorien und Techniken der Repräsentation terminologischen Wissens nützlich. Diese sollten über eine einfache Auflistung ,aller' Konzepte des betrachteten Fachgebiets (der betrachteten Abteilung, des betrachteten Prozesses etc.) hinausgehen. Wie Léon Araúz, Faber und Montero Martínez (2012, 110 ff.) treffend darlegen, sind gerade in einer Terminologie-Datenbank nicht nur die Beschreibungen isolierter Konzepte, sondern ebenso die Verknüpfungen semantisch-konzeptueller Art wichtig.

In der Terminologielehre sind zwar taxonomische Beziehungen geläufig (d. h. die Kodierung von Oberbegriff/Unterbegriff). Die zur Repräsentation von Fachwissen notwendigen semantischen Beziehungen gehen aber über diese TypenBeziehungen weit hinaus. Kognitiv-linguistische Annahmen, wie sie etwa Lakoffs (1987) „Conceptual Metaphor Theory“ und Langackers „Cognitive Grammar“ (1987) zugrunde liegen, so argumentieren Léon Araúz, Faber und Montero Martínez (2012, 112 f.), werden unterstützt durch die Ergebnisse neurowissenschaftlicher Versuche. Diese zeigen, so die Autoren, dass die Verarbeitung von Informationen immer in Relation zu anderen Informationen erfolge. Insbesondere sei die semantische Darstellung nicht von Wahrnehmung, Handlung und Introspektion abtrennbar, und semantische Repräsentationen würden vom Wahrnehmenden selbst und dem Kontext der Wahrnehmung beeinflusst.

There is an increasing consensus in favor of a more dynamic view of cognitive processing or situated cognition, which reflects the assumption that cognition is typically grounded in multiple ways. These include simulations, situated action, and even bodily states. (Léon Araúz/Faber/Montero Martínez 2012, 112)

Eine für die Terminologie besonders relevante Schlussfolgerung, die die Autoren ziehen, bezieht sich direkt auf die semantisch-konzeptuellen Relationen:

[...] knowledge acquisition requires simulation of human interaction with objects, and this signifies that non-hierarchical relations that define the goal, intended purpose, affordances, and result of the manipulation and use of an object [...] are just as important as hierarchical ones, such as type_of or part_of (Léon Araúz/Faber/Montero Martínez 2012,115)

Aus der Perspektive von Fachübersetzern, die auf Termdatenbanken zurückgreifen, bezeichnet Melby (2012) semantische Beziehungen als eine Information, die 
idealerweise in einer „high-end termbase“ vorhanden sein sollte. Diese sowie weitere Informationen wie die Angabe einer Definition und/oder eines Beispielsatzes helfe dem Übersetzer, die jeweils passende Benennung auszuwählen. Melby (2012, 21) führt Beispiele für konzeptuelle Relationen an, darunter auch so genannte associated relations. Neben dem Erstellen und Übersetzen von Texten weist er auf weitere Anwendungsgebiete von Terminologie hin, die von konzeptuellen Relationen profitieren.

Die Frage ist nun, wie diese konzeptuellen Relationen praktisch in einer Terminologiedatenbank hinterlegt werden können. Hilfreich wäre es, sich an existierenden linguistischen und/oder kognitionswissenschaftlichen Theorien zu orientieren, so etwa an der Frame-Semantik. Hierbei ist letztendlich nicht entscheidend, für welche Konzeption von Frames man sich entscheidet. Je nach Umfeld und Umständen der Erstellung, des Darstellungsbereichs und der Anwendung der Terminologiedatenbank kann die Entscheidung unterschiedlich ausfallen. In Abschnitt 3 plädieren wir deswegen für ein hybrides Frame-Modell, das einerseits auf Minskys Konzeption basiert, andererseits aber auch die FrameNet-Datenbank als eine hilfreiche semantische Ressource nutzt. Die notwendigen Voraussetzungen für die Anwendung von Frames in einem Terminologiesystem erläutern wir zunächst im folgenden Abschnitt.

\subsection{Datenmodell in einem Terminologiesystem}

In modernen Terminologiesystemen werden unterschiedliche Datenmodelle verwendet. Das Datenmodell, das wir im Folgenden vorstellen möchten, folgt der Darstellung der Software crossTerm, enthalten im Across Language Server (www.across.net, letzter Zugriff: 28.11.2015). Anderen Systemen liegen ähnliche Modelle zugrunde.

Abb. 2 visualisiert die wichtigsten Entitäten des Datenmodells, nämlich ,Eintrag' (Konzept) und ,Term'. In der Terminologiearbeit bzw. im Zusammenhang mit den Systemen zur Terminologieverwaltung wird ,Eintrag' gleichbedeutend mit ,Konzept' verwendet. Einem terminologischen Eintrag kann eine beliebige Anzahl an Termen $(0 \ldots \mathrm{N})$ in jeder Sprache zugeordnet sein, die auf das repräsentierte Konzept referieren.

Ein Konzept zeichnet sich durch Attribute (wie etwa „Definition“, „Abbildung“, „Fachgebiet") und andere Metadaten aus, die es näher beschreiben. In der 
Terminologiearbeit werden Attribute häufig auch als „Datenkategorien“ bezeichnet. Der Einfachheit halber sind in Abb. 2 alle Attribute gleich dargestellt. Einige der Datenkategorien können für ein Konzept mehrfach verwendet werden, z. B. können mehrere „Definitionen“ und „Abbildungen“ existieren. Während „Definitionen“ textueller Art sind, handelt es sich bei „Abbildungen“ um Daten im Grafik-Format. Für das Attribut „Fachgebiet“ existiert typischerweise eine Werteliste, aus der pro Konzept nur ein Fachgebiet ausgewählt werden kann.

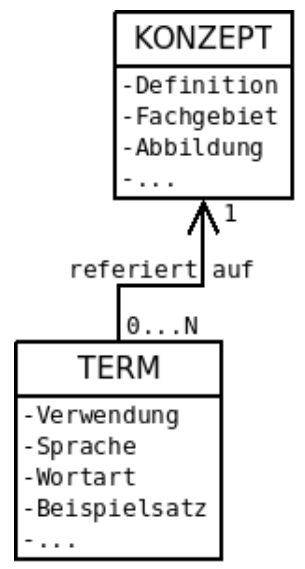

Abb. 2: Datenmodell in einem Terminologiesystem

Der Term ,verfügt' über besondere Kategorien, etwa zu Verwendungshinweisen und zu den verwendeten Sprachen (z. B. Deutsch, Englisch oder jede andere Sprache, in der die Terminologiedatenbank gepflegt wird). Dies dient $u$. a. als Suchfunktion im Terminologiesystem, und es erlaubt auch Zusatzfunktionen, mit denen das Programm Terme in Texten auffindet und gemäß dem präskriptiven Ansatz (vgl. Abschnitt 2.3) gegen die Verwendungshinweise prüft. Zahlreiche andere Datenkategorien, darunter Textfelder für „Beispielsätze“, Auswahllisten für „Wortart“ usw., finden sich ebenfalls hier.

Standardmäßig enthält das hier betrachtete System crossTerm viele Term-Datenkategorien aus einem sogenannten Dialekt des Standardformats „TermBase Exchange“ (TBX) zum Austausch von Terminologiedaten (ISO 30042: 2008). Bei- 
spiele dieser Term-Datenkategorien sind „hyphenation“, „pronunciation“, „grammatical gender“, „etymology“ und „register“. Nicht alle Default-TBX-Datenkategorien sind jedoch von Anfang an aktiviert. Um dies zu illustrieren, ist es nötig, noch einen weiteren Bestandteil des crossTerm-Datenmodells einzuführen, und zwar die so genannte „Vorlage“ (auch „Template“ genannt). Es gibt Vorlagen für Konzepte und Terme. Die Vorlagen bestimmen, welche Datenkategorien beim Anlegen und Pflegen eines Konzepts/Terms ausgefüllt werden.
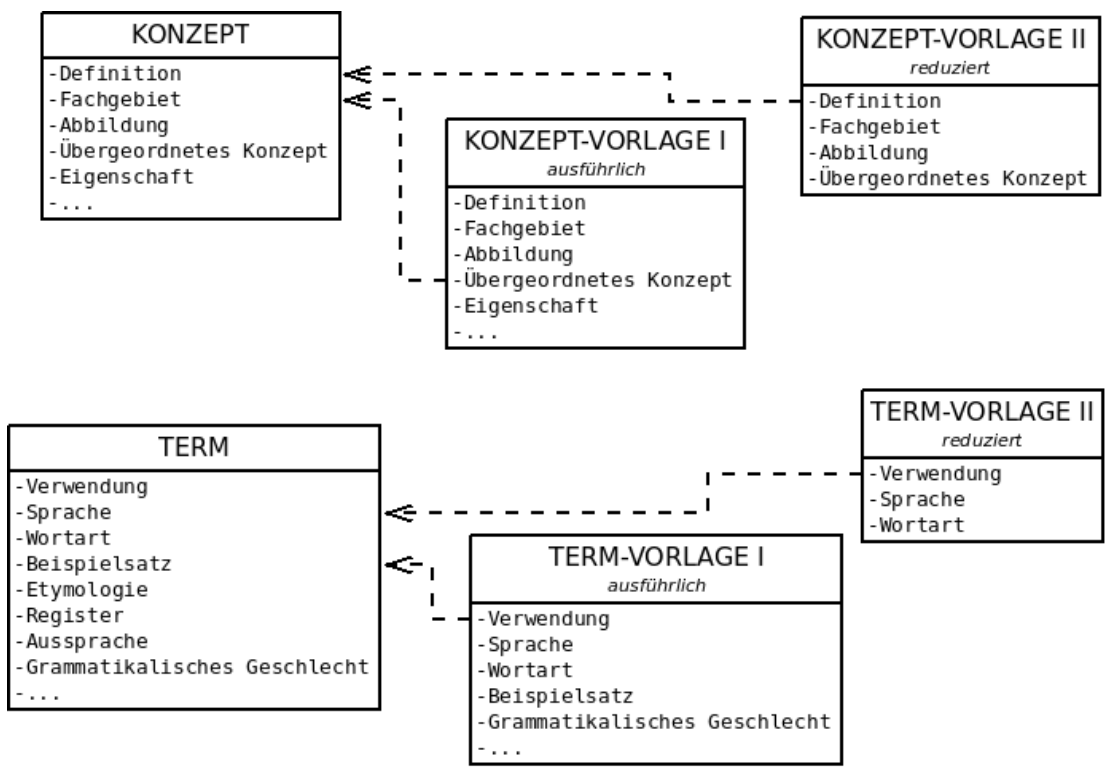

Abb. 3: Vorlagen für Konzepte und Terme

Abb. 3 illustriert dies anhand von je zwei Vorlagen für Konzepte und Terme. Die Konzept-Vorlage I aktiviert alle für die Entität ,Konzept ' vorhandenen Datenkategorien, während die Konzept-Vorlage II nur vier davon enthält. Bei der Anlage und Pflege von Konzepten in der Terminologiedatenbank wählt der Terminologe die Vorlage aus, die aktuell benötigt wird. Auf der Term-Ebene sind einige Datenkategorien in keiner der beiden Vorlagen enthalten („Etymologie“, „Register“, 
„Aussprache“). Sie werden bei Anlage und Pflege von Termen also nie angezeigt und könnten aus der Terminologiedatenbank entfernt werden.

Standardmäßig enthält das Terminologiesystem bereits eine Vorlage mit einer Auswahl von Standard-Datenkategorien, sodass sofort mit der Arbeit begonnen werden kann. Der Terminologe kann aber auch neue Datenkategorien anlegen und diese in ein Template aufnehmen. Ebenso kann er einige oder alle der standardmäßig aktivierten Datenkategorien entfernen (abgesehen von systeminternen Kategorien wie „Sprache“ oder „Verwendungshinweis“). In der fortgeschrittenen Terminologiearbeit ist die Anlage eigener Datenkategorien häufig anzutreffen. Diese Kategorien sind beispielsweise relevant für ein bestimmtes Fachgebiet, für eine bestimmte Firma oder eine bestimmte Abteilung. Ihre Aufnahme in einen Standard wie TBX oder in ein System, das allen Anwendern dienen soll, wäre jedoch sehr aufwendig, und sie ist auch nicht notwendig. Generell kann der Terminologe aber beliebig viele Templates anlegen und diese mit unterschiedlichen Datenkategorien spezifizieren.

\section{Frames und ihre Anwendung in einem Terminologiesystem}

Wie in jüngerer Zeit wiederholt diskutiert (Peterson 2007; Busse 2012; Löbner 2014; Ziem 2013, 2014a, 5-48; vgl. auch Sektion 1 in diesem Band), liegt zwar den modernen Frame-Ansätzen wie dem Minskys (1975; 1986, 221 ff.) im Bereich der Künstlichen Intelligenz-Forschung, dem Barsalous (1992) in den Kognitionswissenschaften bzw. der Kognitiven Psychologie sowie der linguistischen FrameSemantik Fillmores (etwa 1975; 1985), einschließlich ihrer Weiterführung in FrameNet, ein Schema-Begriff zugrunde. Jedoch fällt dessen Modellierung im Detail sehr unterschiedlich aus. Mit Blick auf Anwendungsmöglichkeiten in Terminologiesystemen betreffen Unterschiede nicht zuletzt auch die definitorische Bestimmung der Strukturkonstituenten von Frames selbst. Auf diese möchten wir deshalb zunächst eingehen.

Zur Übertragung auf die terminologische Praxis und auf Einheiten in Systemen zur Terminologieverwaltung bietet sich die aus der Künstlichen IntelligenzForschung (KI) stammende Konzeption Minskys (1975) an. Wie in Tabelle 3 am 
Beispiel des bekannten Apfel-Frames illustriert (Minsky 1986, 221), nimmt ein einfacher KI-Frame die Gestalt einer Attribut-Wert-Matrix an.

\begin{tabular}{ll}
\hline APFEL & \\
\hline Form & rund \\
\hline Farbe & rot \\
\hline Größe & apfelgroß \\
\hline Material & Fruchtfleisch \\
\hline Geschmack & apfelig \\
\hline Struktur & mit dünner Schale \\
\hline
\end{tabular}

Tabelle 3: Apfel-Frame nach Minsky (1986, 204, 221), vgl. Lönneker (2003, 7)

Anhand dieses Beispiels lassen sich die Strukturkonstituenten eines Frames begrifflich etwas genauer bestimmen. Der Apfel-Frame illustriert die in der künstlichen Intelligenz und auch in der linguistischen Semantik bekannten Begriffe des "Slots“ und „Fillers“. Ein Slot ist ein Aspekt des Konzepts, das durch den Frame beschrieben wird. Für den Apfel werden z. B. Form, Farbe, Größe, Material, Geschmack und Struktur als relevante Aspekte bzw. Beschreibungskategorien angenommen. Ein Filler ist der Wert, der für ein Slot für das betrachtete Konzept angegeben wird, also die Antwort auf die Frage, welche Form, Farbe, Größe usw. Äpfel haben. Es kommt vor, dass Kategorien nicht starr beschrieben werden können, sondern Exemplare unterschiedlicher Art enthalten. Daher sind die Filler als die üblicherweise anzutreffenden Werte anzusehen oder auch als diejenigen, die einem menschlichen Betrachter als erste in den Sinn kommen - die typischen Werte oder auch Standardwerte. Minsky $(1975,228)$ spricht hier auch von „default assignments“. Ein bestimmter Apfel könnte demnach auch grün statt rot sein, obwohl der Frame den Standardwert „rot“ für den Slot „Farbe“ vorgibt. Diesem Umstand trägt auch Barsalou (1992) in seinem Frame-Ansatz Rechnung, ohne allerdings Standardwerte gesondert zu berücksichtigen; Ähnliches gilt für Frames, wie sie im Berkeleyer FrameNet-Projekt thematisiert werden (vgl. Ziem 2014b).

Nützlich an Minskys Ansatz ist die Annahme einer prinzipiell flexiblen konzeptuellen Struktur mit rekursiven Eigenschaften. Ein Apfelbauer, der mehrere 
Sorten von Apfelbäumen mit unterschiedlichen Äpfeln anbaut, kann mit denselben Slots etwa alle Apfelsorten beschreiben, ggf. mit leicht unterschiedlichen Standardwerten bei Slots wie „Größe“ und „Farbe“. Falls nötig, kann er für eine bestimmte Apfelsorte weitere Slots hinzufügen, um spezifische Eigenschaften einzubeziehen. Je nach Bedarf und Anwendungszusammenhang können Frames mithin auf verschiedenen Ebene der Granularität angesetzt werden. Das eine Ende der Granularitätsskala bildet dabei eine maximal schematische Repräsentation eines Apfels in Gestalt eines Apfel-Frames ohne Filler. Am anderen Ende befände sich hingegen ein maximal spezifizierter Frame, also die Repräsentation eines einzelnen Exemplars (Tokens, hier: eines konkreten einzelnen Apfels). Auf diese Weise lassen sich Konzepte und Objekte vergleichen oder bestimmte Abläufe wie das Packen von Äpfeln planen, auch per Computerprogramm.

Minsky bleibt zwar hinsichtlich der Frage indifferent, welchen ontologischen Status spezifizierte Slots und Filler haben; wie bereits erwähnt, weist er jedoch darauf hin, dass sich Slots als Fragen verstehen lassen, die sinnvoll mit Bezug auf den Referenten (hier: Äpfel) gestellt werden können. So stellt Minsky $(1975,246)$ fest:

Examinations of linguistic discourse leads [...] to a view of the frame concept in which the "terminals" [i. e. "slots" Anmerkung der Verfasser] serve to represent the questions most likely to arise in a situation. To make this important viewpoint more explicit, I will spell out this reinterpretation: A Frame is a collection of questions to be asked about a hypothetical situation; it specifies issues to be raised and methods to be used in dealing with them.

Vor diesem Hintergrund liegt die Annahme nahe, Filler als Prädikate zu begreifen, die sich einer Bezugsgröße zuschreiben lassen. Sprachlich handelt es sich somit um Prädikationsstrukturen, die sich systematisch rekonstruieren lassen. Ein solches Konzept von Frames als Prädikationsstrukturen erweist sich als kompatibel mit dem Verständnis von Frames als Valenzrahmen, wie es dem Berkeleyer FrameNet-Projekt zugrunde liegt (Ziem 2014b, 282 ff.). Zugleich ist es umfassender und weniger restriktiv, insofern prädikative Zuschreibungen nicht an die Valenz des jeweiligen Zielausdrucks gebunden sind.

Am Beispiel des Apfel-Frames wird eine Herausforderung insbesondere für empirisch-praktische Anwendungsdomänen des Frame-Begriffes sichtbar; sie betrifft die frame-geleitete Erfassung von Weltwissen und besteht unabhängig von 
der gewählten Art der Wissensrepräsentation und -modellierung: Interessanterweise ist es nicht trivial, gerade Gegenstände des Alltags in einer formalisierten Repräsentation zu beschreiben. So entsteht etwa die Tautologie, dass ein Apfel einen apfeligen Geschmack hat und apfelgroß ist. Da wir Menschen alle so vertraut mit Äpfeln sind und sie aus unserer Erfahrung kennen, sind die Filler korrekt und treffend. Ein Leser, Computer oder Roboter, der diese Erfahrung nicht teilt, kann indes aus diesen Angaben keine Erkenntnisse gewinnen. Bei neuen Erfindungen oder fachspezifischen Konzepten, die man jemandem erklären oder in Frames abbilden möchte, sollte man Filler also anders angeben - z. B. für die Größe einen Vergleich (,wie eine Faust“) oder eine Maßeinheit wählen. Wie der KI-Forscher Oren Etzioni (2014) betont, ist es immer noch eine Herausforderung, sich bei der Extraktion und Repräsentation von Wissen zu Konzepten des menschlichen Alltags auf diejenigen Slots und Filler zu konzentrieren, die gerade das grundlegende Wissen beschreiben. Die Gewinnung und Darstellung technischer oder fachspezifischer Einzelheiten und Fakten dagegen scheint wesentlich einfacher (z. B. Nährwerttabelle für $100 \mathrm{~g}$ Apfel). Etzioni (2014) stellt hierzu fest: „Knowledge bases are rich in facts, but knowledge poor.“

In der Terminologiearbeit ist man mit diesem Problem nicht oder nur in geringerer Intensität konfrontiert, da ja gerade Fachwissen oder eine fachwissenschaftliche Sicht auf alltägliche Dinge festgehalten und nahegebracht werden sollen. Außerdem wird in der Terminologieforschung nicht das Ziel verfolgt, die Wissensbestände für KI-Anwendungen (z. B. automatische Beantwortung von Fragen, Reasoning) auswertbar zu machen. Terminologiedatenbanken richten sich vorrangig an den Menschen als Konsumenten, auch wenn bei intensiver Beschäftigung mit der Materie immer wieder eine Annäherung an die Informatik zu beobachten ist (durch Annäherung an Ontologien, Semantic Web und ähnliche Ansätze, siehe z. B. León Araúz et al. 2012, 169-170; Melby 2012, 21).

\subsection{Frames in Terminologiesystemen: ein Beispiel aus der Praxis}

Beim Aufbau von Terminologiesystemen ist der KI-inspirierte Frame-Ansatz durchaus nützlich. Dies lässt sich an einem Beispiel aus der Praxis illustrieren. Terminologieverwaltungssysteme wie crossTerm werden von unterschiedlichen 
Unternehmen eingesetzt, die spezialisierte Produkte vertreiben und entsprechende Dienstleistungen anbieten, z. B. in der Telekommunikation, Medizintechnik, Unterhaltungselektronik, im Maschinen- und Anlagenbau usw. Als Beispiel soll im Folgenden die Verpackungsindustrie dienen, und zwar solche Unternehmen, die Verpackungsmaschinen für kleinere und mittlere Endkunden herstellen.

\begin{tabular}{ll}
\hline ,Kammermaschine & \\
\hline Funktionsweise & $\begin{array}{l}\text { Beladen, Evakuieren von Kammer und Folienbeutel, } \\
\text { Siegeln des Beutels, Belüften der Kammer, Entladen }\end{array}$ \\
\hline Verwendungszweck & $\begin{array}{l}\text { Versiegeln von Konsumgütern und medizinischen Pro- } \\
\text { dukten }\end{array}$ \\
\hline Maschinengröße & klein bis sehr groß \\
\hline Produktionsvolumen & kleine bis große Chargen \\
\hline Bestandteil & Edelstahlgehäuse \\
\cline { 2 - 2 } & Kammer \\
\cline { 2 - 2 } & Steuerung \\
\hline$\ldots$ & $\ldots$. \\
\hline
\end{tabular}

Tabelle 4: Frame-Entwurf für das Konzept ,Kammermaschine‘

In unserem Beispiel möchte der Verpackungsmaschinen-Produzent jene Eigenschaften der Maschine, die sich für den Ablauf und das Ergebnis des Verpackungshergangs als relevant erweisen, terminologisch präzise erfassen. Seine Ziele entsprechen denjenigen, die in Abschnitt 2.2 für die Terminologie allgemein dargestellt wurden: Die Mitarbeiter in Entwicklung, Verkauf und Kundendienst sollen die gleichen Benennungen verwenden und allen soll eine gemeinsame Wissensbasis zur Verfügung stehen, die ein geteiltes grundlegendes Verständnis schafft.

Im Kanon dieser Maschinen befinden sich solche, mit deren Hilfe Ware in Folienbeutel verpackt wird. ${ }^{4}$ Aufgrund ihrer Bau- und Funktionsweise werden die

4 Die Beispiele sind inspiriert durch öffentlich verfügbare Produktbeschreibungen der Firma MULTIVAC (http://www.multivac.com/). Für eventuelle Irrtümer sind allein die Verfasser verantwortlich. 
Maschinen auch als „Kammermaschinen“ oder „Vakuumkammermaschinen“ bezeichnet. In einer Kammer wird ein Beutel mit dem zu verpackendem Gut platziert; in Kammer und Beutel wird ein Vakuum erzeugt, sodass der Beutel eng an der Ware anliegt; der Beutel wird versiegelt; der Druck in der Kammer wird langsam wieder normalisiert, sodass der Beutel unbeschädigt bleibt und herausgenommen werden kann. Der Produzent hat mehrere Maschinenserien im Angebot, vereinzelt fertigt er sogar Sondermodelle an. Die „Funktionsweise“ sowie der „Verwendungszweck“ aller Maschinen in den verschiedenen Serien sind gleich oder ähnlich. Auch weitere Aspekte wie „Maschinengröße“ oder „Produktionsvolumen“ sind immer relevant, können jedoch von Maschine zu Maschine unterschiedliche Ausprägungen haben.

\begin{tabular}{l}
\hline Konzept-Vorlage ,Kammermaschine \\
\hline Funktionsweise \\
\hline Verwendungszweck \\
\hline Maschinengröße \\
\hline Produktionsvolumen \\
\hline Bestandteil(e) \\
\hline$\ldots$ \\
\hline
\end{tabular}

Tabelle 5: Vorlage zum Konzept ,Kammermaschine`

In diesem Beispiel besteht die Aufgabe des Terminologen dieses Unternehmens darin, für das Konzept ,Kammermaschine' einen Frame mit einem Set an Slots zu entwerfen, etwa ähnlich dem in Tabelle 4 dargestellten Konzept. Für alle bestehenden oder in Zukunft neu erfundenen Maschinen(serien) wird der Frame in der Terminologie wiederverwendet und nur die Filler werden entsprechend eingetragen. Hierzu kann der Terminologe auf ein Terminologiesystem zurückgreifen. Wie bereits erwähnt, bieten moderne Terminologietools die Möglichkeit, Datenkategorien auf Konzept- und Term-Ebene anzulegen. Die Datenkategorien für Konzepte entsprechen den Slots des KI-Frames bzw. in FrameNet Frame-Elementen oder, allgemeiner, Prädikationsleerstellen. Der Terminologe legt also die Datenkategorien „Funktionsweise“, „Verwendungszweck“, „Maschinengröße“, 
„Produktionsvolumen“ und ggf. weitere an und fügt diese einer Konzept-Vorlage für ,Kammermaschine“ hinzu. Diese Vorlage wird bei der Anlage einer neuen Maschine in der Terminologiedatenbank wiederverwendet und mit Werten spezifiziert. Außerdem fügt der Terminologe den Konzepten die Benennungen bzw. Terme in einer oder mehreren Sprachen hinzu. Zur Betrachtung der Terme und ihrer Eigenschaften trägt der KI-Frame hingegen nichts Wesentliches bei.

Ein leistungsstarkes Terminologietool bietet zudem die Möglichkeit, für eine beliebige Anzahl allgemeiner Konzepte bzw. Frames Vorlagen anzulegen (für ,Kammermaschine‘ vgl. Tabelle 5). Auf diese Strukturen kann der Terminologe bei der Anlage weniger allgemeiner Frames zurückgreifen, z. B. ,Tischkammermaschine' (Kammermaschinen, die auf einem Tisch stehen können) vs. ,Standkammermaschine' (vgl. Tabelle 6).

\begin{tabular}{|c|c|c|}
\hline & ,Tischkammermaschine“ & ,Standkammermaschine‘ \\
\hline Funktionsweise & $\begin{array}{l}\text { Beladen, Evakuieren von Kam- } \\
\text { mer und Folienbeutel, Siegeln } \\
\text { des Beutels, Belüften der } \\
\text { Kammer, Entladen }\end{array}$ & $\begin{array}{l}\text { Beladen, Evakuieren von Kam- } \\
\text { mer und Folienbeutel, Siegeln } \\
\text { des Beutels, Belüften der } \\
\text { Kammer, Entladen }\end{array}$ \\
\hline $\begin{array}{l}\text { Verwendungs- } \\
\text { zweck }\end{array}$ & $\begin{array}{l}\text { Versiegeln von Konsumgütern } \\
\text { und medizinischen Produkten }\end{array}$ & $\begin{array}{l}\text { Versiegeln von Konsumgütern } \\
\text { und medizinischen Produkten }\end{array}$ \\
\hline $\begin{array}{l}\text { Maschinen- } \\
\text { größe }\end{array}$ & sehr klein & klein bis sehr groß \\
\hline $\begin{array}{l}\text { Produktionsvo- } \\
\text { lumen }\end{array}$ & kleine Chargen & kleinere bis mittlere Chargen \\
\hline \multirow[t]{3}{*}{ Bestandteil(e) } & Gehäuse & Edelstahlgehäuse \\
\hline & Kammer & Kammer \\
\hline & Steuerung & Steuerung \\
\hline Zielgruppe & $\begin{array}{l}\text { Metzgereien, Hotels, Restau- } \\
\text { rants etc. }\end{array}$ & $\begin{array}{l}\text { kleine bis mittelgroße Unter- } \\
\text { nehmen v. a. der Lebensmit- } \\
\text { telindustrie }\end{array}$ \\
\hline
\end{tabular}

Tabelle 6: Konzepte ,Tischkammermaschine‘ und ,Standkammermaschine“ im Vergleich

Die Verwendung der Vorlage stellt sicher, dass dem Terminologen die für wichtig erachteten Datenkategorien bei der Anlage eines Konzepts sofort zur Verfügung 
stehen. Die erneute Arbeit der Auswahl bzw. Erstellung von Datenkategorien, die für diese Art von Maschinen sinnvoll sind, erübrigt sich. Wenn diese Datenkategorien bei jeder Maschine vorhanden sind, erleichtert dies auch beim Nachschlagen in der Terminologiedatenbank dem lesenden Benutzer das Auffinden und Vergleichen von Informationen.

Wünschenswert wäre im Terminologiesystem die Anlage von Standardwerten per Template und eine Hierarchisierung der Templates, um z. B. die Filler „Gehäuse“, „Kammer“ und „Versiegeln von Konsumgütern und medizinischen Produkten" bei Wiederverwendung des Templates zu erhalten bzw. zu vererben. Diese Funktionen bieten aber trotz der Wichtigkeit hierarchischer Beziehungen zurzeit selbst High-End-Tools im Terminologiebereich üblicherweise (noch) nicht an. Hierarchische und andere Beziehungen können jedoch in diesen Systemen über Verweise dargestellt werden.

\subsection{Beschreibung der Slots: von Minsky zum FrameNet}

Der Apfel-Frame (Tabelle 3) lässt sich insbesondere deshalb intuitiv erschließen, weil uns das Konzept Apfel sehr vertraut ist. Das Konzept „Kammermaschine“ dürfte dagegen weniger bekannt sein; Gleiches gilt für viele andere Konzepte aus speziellen Fachgebieten, denen sich die Terminologiearbeit widmet.

Daher wird in der Terminologiearbeit - ebenso wie in der linguistischen Frame-Semantik im Anschluss an FrameNet (vgl. hierzu exemplarisch Fillmore/Baker 2010; zum so genannten EcoLexicon vgl. Faber 2012) - mit weiteren Kategorien wie „Definition“, „Abbildung“ und „Beispielsatz“ gearbeitet. Diese lassen sich ebenfalls in den Frame als Slots bzw. in das terminologische Konzept als Datenkategorien aufnehmen. Allgemeingültige Datenkategorien wie z. B. „Definition“ sollten in jedem Konzept enthalten sein; in den Terminologietools befinden sie sich üblicherweise in der Standard-Vorlage eines terminologischen Konzepts. Die Definition trägt dazu bei, dass die Slots eines Frames besser verstanden werden. León Araúz et al. (2012, 148 ff.) weisen darauf hin, dass gute terminologische Definitionen selbst anhand von Templates aufgebaut sind, die wie ein Mini-Frame aussehen.

Dass Minsky $(1975 ; 1986)$ weder die einzelnen Slots näher beschreibt und definiert noch eine Systematik zur Ermittlung von Slots anbietet, schränkt den Nutzen seines Frame-Konzepts für die Entwicklung von Terminologiesystemen ein. 
Nötig ist zumindest, Slots konsistent und in einer für Ersteller und Nutzer eines Terminologiesystems verständlichen Weise zu beschreiben. Ebenso wäre es hilfreich und nützlich (und aus grundlagentheoretischer Sicht auch erforderlich) auszuweisen, wie Slots bzw. Frame-Elemente systematisch und möglichst exhaustiv ermittelt werden können. Aus Sicht des Praktikers wäre es schließlich wünschenswert, für beliebige Entitäten ohne großen Aufwand Frames mit dem Zweck zu kreieren, die Kommunikation über diese Entitäten zu vereinfachen und zu optimieren. Die einzige umfassende Datenbank, die für diesen Zweck bislang bereitsteht, ist FrameNet. Mit über 13.000 lexikalischen Einheiten, also Wort-Bedeutungspaaren, die semantisch mithilfe von derzeit ungefähr 1.200 Frames erfasst werden (Stand: Dezember 2015), deckt diese lexikalisch-semantische Datenbank grob den Alltagswortschatz ab.

Wie lässt sich nun FrameNet zum Aufbau von Terminologiesystemen nutzen? In seinen frühen Arbeiten, die der Entwicklung einer so genannten Kasusgrammatik verpflichtet sind (Fillmore 1968), schlägt Fillmore ein relativ kleines Set an Kategorien, sogenannten „Tiefenkasus“ („deep cases“), vor, denen er einen universellen Status zuschreibt: „a set of universal, presumably innate, concepts which identify certain types of judgments human beings are capable of making about [...] events“. Zu solchen tiefensemantischen Rollen gehören beispielsweise „Instrumental“ und „Factitive“; erstere definiert er als „the inanimate force or object causally involved in the action or state identified by the verb", letztere als "the object or being resulting from the action or state identified by the verb [...]" (Fillmore 1968, 46). Nicht zuletzt aufgrund der empirischen Schwierigkeit, die im Datenmaterial attestierte Vielzahl und Vielgestaltigkeit realisierter thematischer Rollen auf ein Set von einigen wenigen Tiefenkasus zu reduzieren (Fillmore 2006, 616), rückt Fillmore jedoch von der problematischen Annahme eines geschlossenen, universellen Sets semantisch-thematischer Rollen ab. Im Zuge seiner frühen framesemantischen Arbeiten entwickelt er stattdessen seit Mitte der 1970er Jahre ein Konzept von semantischer und syntaktischer Valenz, das auch dem FrameNet-Projekt zugrunde liegt. Frames werden hier definiert durch Frame-Ele- 
mente, d. h. durch empirisch ermittelte semantische Rollen, die zum Valenzrahmen des jeweiligen Zielausdruckes gehören. ${ }^{5}$ Der entscheidende Unterschied zum kasusgrammatischen Konzept des Tiefenkasus besteht darin, dass FrameElemente jeweils relativ zu dem Frame, den sie näher bestimmen, definiert werden. Die Menge der Frame-Elemente ist infolgedessen nicht festgeschrieben; vielmehr handelt es sich schon deshalb um ein offenes Set, weil jeder Frame auch Frame-Elemente umfassen kann, die nur für diesen Frame spezifisch sind, also nicht von einem übergeordneten Frame ererbt werden.

Für das eingeführte Beispiel des maschinellen Packens ist der so genannte Placing-Frame relevant und - auch in praktischer Hinsicht - nützlich; ${ }^{6}$ er wird unter anderem von den Lexikalischen Einheiten packen und Paket aufgerufen. In FrameNet ist dieser Frame folgendermaßen definiert: ${ }^{7}$

Generally without overall (translational) motion, an Agent places a THEME at a location, the GOAL, which is profiled. In this frame, the THEME is under the control of the AgENT/CAUSE at the time of its arrival at the GOAL.

[AgentDavid] placed [Themehis briefcase] [Goalon the floor]. This frame differs from Filling in that it focuses on the THEME rather than the effect on the GoAL entity. It differs from Removing in focusing on the GoAL rather than the SOURCE of motion for the THEME.

Jede Definition enthält einen annotierten Beispielsatz. Im angeführten Beispielsatz wird der Frame durch das Wort placed aufgerufen, wobei die Frame-Elemente AGENT, THEME und GOAL instantiiert sind. Die entsprechenden Phrasen werden, wie im Beispiel geschehen, gekennzeichnet und semantisch annotiert. Die Definition enthält auch Hinweise zur Abgrenzung des P lacing-Frames von anderen Frames, insbesondere von Filling und Removing. Zudem wird jedes FrameElement definiert, GOAL etwa folgendermaßen: „The FE GOAL is the location where the THEME ends up."

5 Frame-Elemente weisen ähnliche Charakteristika wie Slots eines KI-Frames auf: Auch sie können zwischen verwandten Frames vererbt oder weiterbenutzt werden. $\mathrm{Zu}$ den Unterschieden und Gemeinsamkeiten vgl. zusammenfassend Busse 2012, 553 ff.; speziell zum FrameNet-Datenmodell vgl. z. B. Lönneker-Rodman/Baker (2009), Ruppenhofer et al. (2010) und Ziem (2014b).

6 Zum Zwecke der besseren Lesbarkeit folgen wir fortan der gängigen Konvention, Frame-Namen durch den Schrifttyp Courier New und Frame-Elemente durch KAPITÄLCHEN typografisch kenntlich zu machen.

7 Vgl. https://framenet.icsi.berkeley.edu/fndrupal/frameIndex, letzter Zugriff: 30.9.2015. 


\subsection{Beziehungen zwischen Frames}

Frames existieren nicht als isolierte Einheiten, sie unterhalten vielmehr vielfältige Beziehungen zu anderen Frames, die in FrameNet ausgewiesen und durch den so genannten FrameGrapher grafisch veranschaulicht werden können. ${ }^{8}$

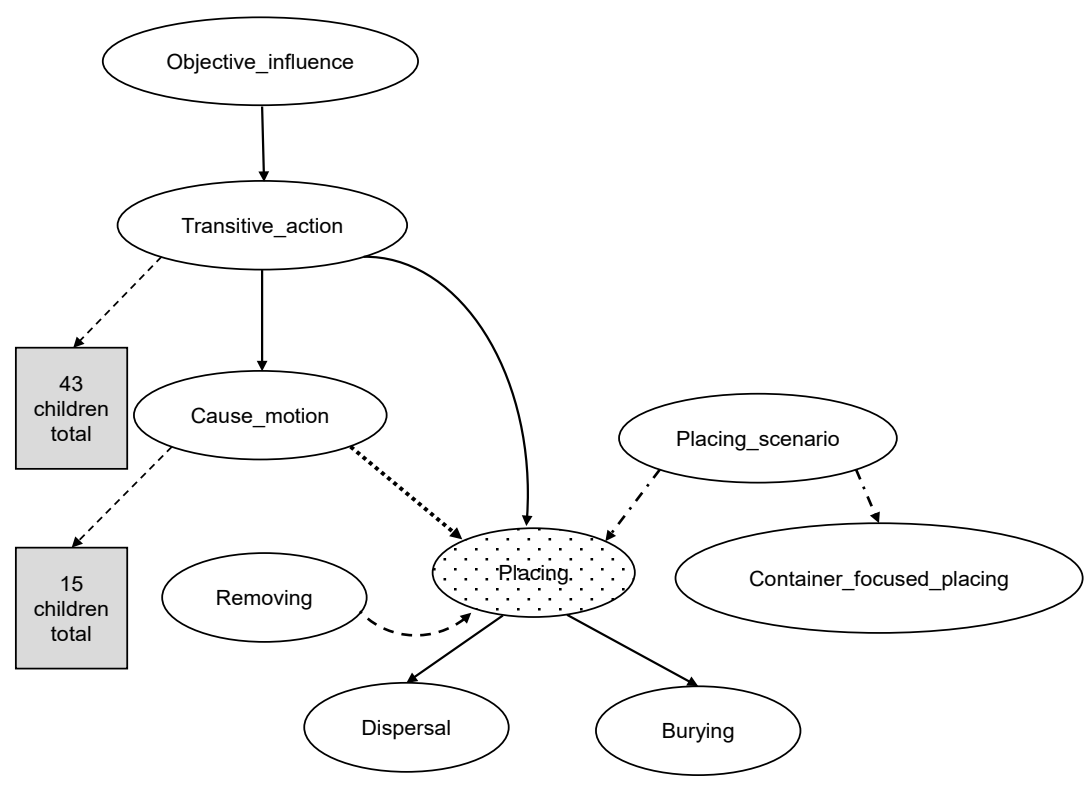

Abb. 4: Frame-zu-Frame-Beziehungen am Beispiel des Placing-Frames ${ }^{9}$

Fillmore und Baker $(2010,330)$ differenzieren zwischen insgesamt sieben Typen von Frame-zu-Frame-Beziehungen. ${ }^{10}$ Der Typ der Beziehung legt jeweils fest, in

8 Vgl. https://framenet.icsi.berkeley.edu/fndrupal/FrameGrapher; letzter Zugriff: 30.10.2015.

9 Abb. 4 zeigt Beziehungen, die der Placing-Frame zu benachbarten Frames unterhält. Aus darstellungstechnischen Gründen beschränkt sich die Illustration auf folgende grundlegende Typen von Beziehungen: (a) Vererbung (dargestellt durch einen Pfeil mit durchgezogener Linie), (b) Subframe (abwechselnd gestrichelte und gepunktete Linie), (c) Perspektive (gepunktete Linie) und (d) Eltern-Kind-Beziehung (gestrichelte Linie).

${ }^{10}$ Diese lassen sich Fillmore und Baker zufolge in drei Gruppen zusammenfassen: (1) Generalisierungs-Relationen (,Vererbung', ,Perspektive', ,brauchen' [engl. ,using']), (2) Ereignisstruktur-Relationen (,Subframes', ,vorangehen') und (3) systematische Relationen (,ursächlich für', ,ausgehen von' [,inchoative of ]). 
welchem semantischen Zusammenhang Frames miteinander stehen. Der Placing-Frame erbt beispielsweise Frame-Elemente vom Transitive_actionFrame, ist zugleich ein Subframe vom Cause_motion-Frame und wirft eine spezifische Perspektive auf das übergeordnete Placing_scenario. Einige der Frame-zu-Frame-Beziehungen, die der Placing-Frame mit anderen Frames unterhält, illustriert Abb. 4.

Frame-zu-Frame-Beziehungen sind für den Aufbau von Terminologiesystem von großem Nutzen, wie sich am Beispiel einer ausführlichen Fallstudie zum Fachgebiet Umwelt zeigen lässt, aus der das frame-basierte Fachlexikon „EcoLexicon" hervorgegangen ist (León Araúz et al. 2012). Im EcoLexikon spielen Relationen zwischen Konzepten eine zentrale Rolle. Die Autoren ermitteln zunächst für ihr Fachgebiet relevante Relationen, so etwa neben hierarchischen und TeilGanzes-Beziehungen auch Zusammenhänge (wie „result_of", „affects“, oder „has_function"). Damit sowohl der Lexikonersteller als auch der Lexikonnutzer weiß, wie diese Relationen zu verstehen sind, werden diese definiert und durch Beispiele, teilweise auch mit Abbildungen, illustriert (León Araúz et al. 2012, 130 ff.). Die Definition der Relation causes umfasst beispielsweise neben fachgebietsspezifischen Beispielen unter anderem auch eine Abgrenzung von anderen relevanten Relationen:

The causes relation links entities and events. For example, WATER causes EROSION. Even though this relation initially seems to be the inverse of result_of, there is a difference that stems from the active role played by certain entities. More specifically, the relation causes only describes the beginning of a process, whereas result_of may link events or entities that are the final consequence of another event. For example, a HURRICANE causes FLOODS during its passage over land, but floods are not the final (and more permanent) result of a hurricane. (León Araúz et al. 2012, 138)

Auf dem Gebiet der Terminologie beschreibt der TBX-Standard (ISO 30042: 2008) ebenfalls einige Datenkategorien bzw. Slots, die sich auf viele Konzepte (bzw. Frames) anwenden lassen. Ein Beispiel dafür ist die Datenkategorie „superordinateConceptGeneric", eine hierarchische Beziehung, vergleichbar der semantischen Hyperonymie- bzw. Hyponomie-Relation. Der Filler fungiert dabei als übergeordnetes Konzept des beschriebenen Frames, er steht also in einer hyperonymischen Relation zu letzterem. Ein anderes Beispiel ist die Datenkategorie 
"subordinateConceptPartitive“, also eine Teil-Ganzes-Beziehung; der Frame repräsentiert hier das Ganze, der Filler eines Slots bildet einen Teil des Ganzen.

In der terminologischen Praxis ist die Verwendung derartiger Slots jedoch nicht sehr weit verbreitet. Abgesehen von Definitionen und Abbildungen, die einen allgemein angesehenen Status in der Terminologiearbeit haben, finden sich in der Praxis selten Datenkategorien auf der Konzeptebene. Konzeptuelle Darstellungen, wie exemplarisch in den Tabellen 5 und 6 für das Beispiel Kammermaschinen veranschaulicht, sind also bislang in der Terminologiearbeit selten anzutreffen. Über die Gründe kann man nur spekulieren. Ein Grund kann die weder sehr ausführliche noch besonders einprägsame Erläuterung der semantischen Relationen im TBX-Standard sein. Ein weiterer Grund könnte darin liegen, dass sich die Terminologiearbeit auf die Beschreibung der Terme (Benennungen und deren Attribute wie Wortart, grammatikalisches Geschlecht, Register usw.) konzentriert und der Beschreibung des Konzepts selbst nur wenig Zeit und Aufmerksamkeit gewidmet werden kann. Dies ist bedauerlich, da gerade die konzeptuellen Informationen und Relationen dem Erfassen und Weitergeben von Informationen über das Fachgebiet dienlich sind (León Araúz et al. 2012, 111 ff.).

Bereits das Ermitteln der relevanten Datenkategorien und ihrer Definitionen trägt zum Verständnis des Fachgebiets und der zu erfassenden Informationen bei. Sinnvoll ist es daher, die Definitionen dieser Datenkategorien ebenfalls im Terminologiesystem zu hinterlegen, insbesondere bei größeren Terminologiebeständen, längeren Terminologieprojekten und/oder der Bearbeitung durch mehrere Personen. Falls das Terminologieverwaltungssystem keine Annotation der Datenkategorien unterstützt, bestünde eine Alternative darin, für jede Datenkategorie ein eigenes Konzept anzulegen und dieses zu definieren. In der Definition der Konzepte eines Fachgebiets wäre es hilfreich, analog zu FrameNet-Definitionen die Bezeichnungen der definierten Datenkategorien zu berücksichtigen. Diese kann der Terminologe auch mit Verweisen versehen, sodass der Benutzer der Terminologiedatenbank weitere Informationen zu den Datenkategorien über Links direkt abrufen kann. 


\section{Aufbau terminologischer Netzwerke und deren grafische Darstellung}

Im Folgenden soll anhand weiterer Beispiele erläutert werden, welchen Nutzen terminologische Netzwerke für die Praxis haben. Insbesondere geht es darum aufzuzeigen, wie sich konzeptuelle Relationen zwischen Frames grafisch darstellen lassen.

Auf konzeptueller Ebene sind Slots (bzw. Datenkategorien) dann von großer Nützlichkeit, wenn Konzepte mittels semantischer Relationen miteinander verbunden werden und so zum Aufbau gedanklicher Netze beitragen können (vgl. die FrameNet-Datenbank und das in Faber 2012 beschriebene EcoLexicon). Viele Terminologieverwaltungssysteme erlauben es aus diesem Grund, Verweise bzw. Links anzulegen, mit denen sich (mehr oder weniger eindeutige) visuelle Repräsentationen von Netzwerken erzeugen lassen. Da es in den Terminologiesystemen kaum formale Beschränkungen für die Angabe von Fillern bzw. Werten gibt, ist zwischen zwei Typen von Verweisen zu unterscheiden. Es finden sich einerseits solche Verweise, die den kompletten Filler betreffen und somit die Beziehung zwischen zwei in der Terminologiedatenbank erfassten Konzepten herstellen, und andererseits solche, die nur Teile des Fillers abdecken. Letztere werden in der Praxis typischerweise innerhalb von längeren Fillern verwendet, also z. B. in Definitionen, Anmerkungen zu Wartung oder Ähnlichem.

Der Unterschied lässt sich anhand von Beispielen verdeutlichen. Im Frame Tischkammermaschine (Tabelle 6) ist etwa als Filler des Slots „Bestandteil(e)“ die Angabe „Gehäuse“ eingetragen. Diese Angabe, in diesem Fall also nur das Wort „Gehäuse“, kann mit einem Verweis hinterlegt werden, der zum Frame Gehäuse in derselben Terminologiedatenbank führt. Dieser Frame wiederum beschreibt, was im Fachgebiet der Kammermaschinen unter einem „Gehäuse“ verstanden wird. Eine derartige Verlinkung kann für alle drei (oder mehr) getrennt angelegten Werte der Datenkategorie „Bestandteil(e)“ vorgenommen werden.

Betrachten wir ein weiteres Beispiel. Im Frame Tischkammermaschine (Tabelle 6) ist als Filler des Slots „Verwendungszweck“ „Versiegeln von Konsumgütern und medizinischen Produkten“ angegeben. In diesem Fall wäre es zwar ebenfalls möglich, die gesamte Angabe mit einem Link zu hinterlegen, der dann auf den Frame Versiegeln von Konsumgütern und medizinischen 
Produkten verweisen könnte. Ein solcher Frame wäre jedoch in kognitiver und funktionaler Hinsicht, also mit Blick auf die Zielvorgabe, Wissen effizient und präzise zu vermitteln, zweifelhaft. Gegebenenfalls unterscheiden sich die Konzepte ,Versiegeln von Konsumgütern' und ,Versiegeln medizinischer Produkte', sodass infolgedessen mindestens zwei Frames benötigt würden. Weiter sollte der Terminologe der Tatsache Rechnung tragen, dass mit den Maschinen noch andere Produkte versiegelt werden könnten. Es ist somit naheliegend, einen Frame für ,Versiegeln“ sowie Frames für ,Konsumgüter' (oder ,Konsumgut') und ,Medizinisches Produkt` anzulegen. Demzufolge verweist der Filler zum Slot „Verwendungszweck" nun wie folgt auf drei Frames:

(1) Verwendungszweck: Versiegeln von Konsumgütern und medizinischen Produkten

Alle Verweise befinden sich innerhalb desselben Fillers (hier: dem Wert der Datenkategorie „Verwendungszweck“). Der erste Link verweist auf den Frame Versiegeln, der zweite auf den Frame Konsumgut, der dritte auf den Frame Medizinisches Produkt. Diese Verweise werden manuell angelegt, wobei eine Wortform wie „Konsumgütern“ ohne weiteres mit einem Verweis zum Konzept ,Konsumgut' versehen werden könnte.

Semantische Informationen, auch solche in Gestalt eines Frames, werden oft als Graph visualisiert, um die Rezeption und das Verständnis zu erleichtern oder die textuelle Ansicht durch eine diagrammatische zu ergänzen. Darüber hinaus werden grafische Darstellungen als Mittel genutzt, um komplexe Netzwerkstrukturen mit einem möglichst hohen Grad an Präzision und Eindeutigkeit zu visualisieren, was in sprachlicher Form nur schwer und nur mit großem Aufwand erreichbar wäre. Dieser Zweck motiviert etwa Visualisierungen im Düsseldorfer Frame-Ansatz (vgl. hierzu Vosgerau/Zielasek/Soom in diesem Band; Wulf in diesem Band). Der bereits erwähnte FrameGrapher des FrameNet-Projekts folgt dagegen primär einem Illustrationszweck. Analoges gilt für die ThinkMap-Ansichten im EcoLexicon. Ähnlich dem FrameGrapher, der es ermöglicht, Relationen zwischen Frames sowie zwischen ihren Frame-Elementen (Slots) zu zeigen, gehen auch EcoLexicon-Darstellungen von Frames aus. Dargestellt werden Relationen, die zwischen einem Frame und seinen Nachbarframes bestehen. ${ }^{11}$ Interaktive

\footnotetext{
11 http://ecolexicon.ugr.es/visual/index_en.html, zuletzt aufgerufen am 20. Oktober 2015.
} 
Darstellungen erlauben es, die angezeigten Relationen einzuschränken sowie anhand der ausgewiesenen Relationen von einem Frame zu einem anderen zu navigieren.

Sobald die Konzepte in einer Terminologie-Datenbank als Frames aufgefasst und entsprechende konzeptuelle Relationen identifiziert, definiert und implementiert worden sind, ist eine grafische Darstellung dieser Relationen in Terminologie-Systemen naheliegend. Die Navigation zwischen Konzepten kann dabei auf den Verweisen basieren. Da die meisten Terminologieverwaltungssysteme derzeit grafische Darstellungen von Beziehungen zwischen Konzepten allenfalls rudimentär unterstützen, sind die folgenden Ausführungen eher als Anregungen für mögliche Weiterentwicklungen der Systeme zu verstehen.

Für den Anwendungsbereich der Terminologie lässt sich die Möglichkeit grafischer Visualisierungen folgendermaßen konkretisieren. Als Beispiel sollen die Slots „Bestandteil(e)“ und „Zweck“ im Frame Tischkammerma schine dienen. Der Slot bzw. die Datenkategorie „Bestandteil(e)“ wurde in der hier exemplarisch behandelten Terminologiedatenbank mehrfach verwendet, da eine Tischkammermaschine mehrere für den betrachteten Bereich relevante Bestandteile hat. Alle Bestandteile sind ebenfalls als Konzepte in der Terminologiedatenbank dargestellt, sodass der Terminologe die Filler mit Verweisen auf diese Konzepte bzw. die sie strukturierende Frames wie in (2) hinterlegt hat.

(2) Bestandteil: Gehäuse

Bestandteil: Steuerung

Bestandteil: Kammer

Der Slot „Verwendungszweck“ ist wie in Beispiel (1) mit einem ausführlicheren Wert versehen, der Verweise auf weitere Frames enthält, siehe Beispiel (3).

(3) Verwendungszweck: Versiegeln von Konsumgütern und medizinischen Produkten

Für die Verweisziele kann bereits in der textuellen Darstellung eine Anzeige bzw. Voransicht generiert werden, z. B. in Form eines Tooltips. Der Inhalt des Tooltips könnte die Definition des verbundenen Konzepts sein. Im nachfolgenden Beispiel (4) werden die Verweisziele in eckigen Klammern dargestellt, um das interne Wissen des Tools über die Verweise zu verdeutlichen; für eine tatsächliche Darstellung soll dies keine Empfehlung sein. 
(4) Verwendungszweck: Versiegeln [VERSIEGELN] von Konsumgütern [KONSUMGUT] und medizinischen Produkten [MEDIZINISCHES PRODUKT]

Das Konzept, seine Slots (Datenkategorien) und die in den Fillern als Verweisziele hinterlegten weiteren Konzepten könnten nun als Datengrundlage für die Erzeugung einer visuellen Repräsentation verwendet werden. Einen solchen Graphen zeigt Abb. 5 ausgehend vom Frame Tischkammermaschine und dessen Slots „Bestandteil“ und „Verwendungszweck“.

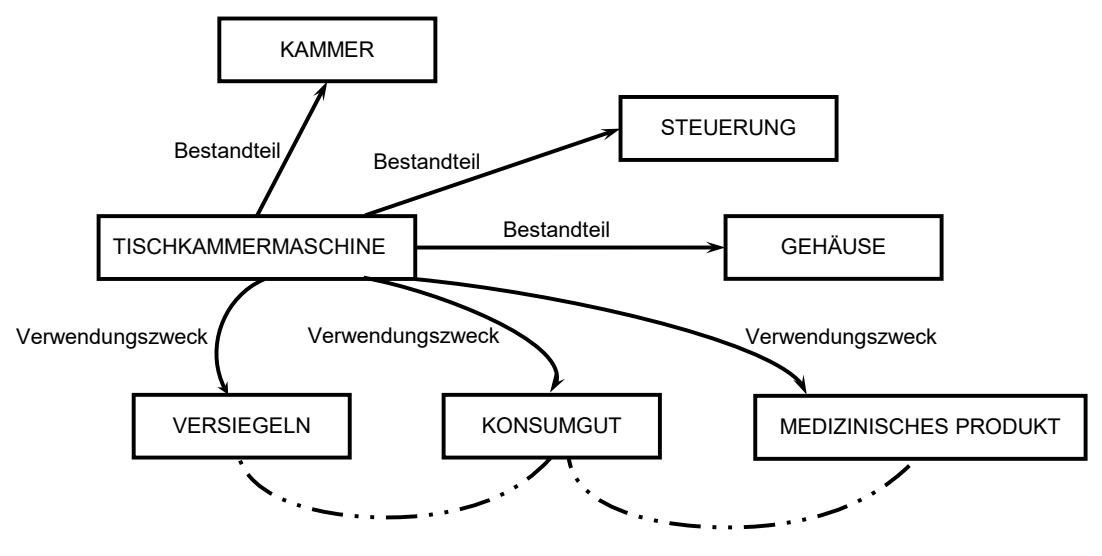

Abb. 5: Beziehungen zwischen Frames (am Beispiel von Tischkammermaschine)

Während die Darstellung der Datenkategorie „Bestandteil“ und ihrer angenommenen Werte unproblematisch ist, da alle Relationen gleichwertig und voneinander unabhängig sind, enthält dagegen der Filler des Slots „Verwendungszweck“ mehrere Links, die untereinander in einer zunächst nicht weiter bekannten Beziehung stehen. Hierfür sind mehrere Darstellungsformen denkbar. In Abb. 5 wird der Zusammenhang durch die gleiche Beschriftung der Achsen mit dem Slot (,Verwendungszweck“) und durch zusätzliche Verbindungslinien zwischen den drei relatierten Frames dargestellt. ${ }^{12}$

12 Anders als im Düsseldorfer Frame-Ansatz handelt es sich bei den Kanten, also den Beziehungen zwischen Frames, nicht um funktionale Relationen (im mathematischen Sinne); vielmehr handelt es sich um Abstraktionsgrößen, die aus dem Datenmaterial gewonnen sind. 
Ohne den Filler selbst semantisch zu parsen, d. h. in seine semantischen Bestandteile und deren Beziehungen zu zerlegen, müssen alle als Verweise gekennzeichneten Bestandteile („Versiegeln“, „Konsumgütern“, „medizinischen Produkten“) bzw. die angegebenen Verweisziele Versiegeln, Konsumgut und medizinisches Produkt als gleichwertig angenommen werden. Insofern erweist sich eine visuelle Repräsentation solcher Slots ohne weitere Analysen zwangsläufig als unpräzise. In einer Terminologiedatenbank werden längere und syntaktisch und semantisch komplexere Werte von Datenkategorien häufig anzutreffen sein, z. B. in Fillern von Slots wie „Definition“, „Anmerkung“, ,Verwendungszweck“, „Zielgruppe“, „Vertragsbedingungen“ usw. Dies ist so lange der Fall, wie die Terminologie für menschliche Nutzer (und nicht für die maschinelle Weiterverarbeitung) bestimmt ist.

Eine Möglichkeit, im vorliegenden Fall die Ungenauigkeit der Visualisierung aufzuheben, besteht in der Aufspaltung des Slots. Der Verwendungszweck einer Verpackungsmaschine kann immer als „Verpacken“ angenommen werden. Im speziellen Beispiel der Tischkammermaschine handelt es sich um eine besondere Art des Verpackens, nämlich des „Versiegelns“. Daher kann dies entweder weiterhin als „Verwendungszweck“ angegeben werden; alternativ wäre die Ergänzung einer speziellen Datenkategorie, hier etwa „Verpackungsart“, möglich, wenn Verpackungsmaschinen in der Terminologiedatenbank besonders prominent und vielfältig sind. Hinzu kommt eine weitere Datenkategorie, z. B. Verpacktes Gut, um weitere Informationen aus Beispiel (3) darzustellen (vgl. hierzu auch das Frame-Element Goods des Placing-Frames in FrameNet). Die Slots „Bestandteil(e)“ und „Zweck“ des Frames Tischkammermaschine könnten in der Terminologiedatenbank somit wie in (5) illustriert repräsentiert werden.

(5) Bestandteil: Gehäuse

Bestandteil: Steuerung

Bestandteil: Kammer

Verwendungszweck: Versiegeln

Verpacktes Gut: Konsumgut

Verpacktes Gut: Medizinisches Produkt

Verloren geht zwar die Darstellung der Beziehung zwischen Versiegeln, Konsumgut und medizinisches Produkt; sie ist von einer anderen Art als 
z. B. die Relation zwischen Gehäuse und medizinisches Produkt. Die Verweise sind aber vollständig durch die Filler abgedeckt. Sie werden von den Terminologen üblicherweise manuell gesetzt, wobei das Terminologietool Unterstützung leistet, so zum Beispiel durch eine Anzeige, ob der Verweis ein gültiges Ziel hat (d. h., ob der gewünschte Zielframe in der Terminologie existiert und korrekt angegeben ist). Die Tools ermöglichen auch, genaue bzw. disambiguierte Verweise zu definieren. Dies bedeutet, dass der Terminologe bei der Existenz von zwei verschiedenen Konzepten mit dem gleichen Term (z. B. Gehäuse) das im jeweiligen Frame- und Fillerkontext passende Konzept als Ziel auswählen kann. Wie erwähnt, ist in einer Terminologiedatenbank ein expliziter und eindeutiger Konzeptname nicht vorgesehen. Das Ziel des Verweises wird daher z. B. anhand der Definition und der im Konzept enthaltenen Terme ausgewählt und kann systemintern mit der eindeutigen Nummer (ID) des Konzepts versehen werden, sodass der Verweis eindeutig ist.

Um die Lesbarkeit der Filler zu vereinfachen (bzw. Flexibilität beim Formulieren längerer Fillertexte zu ermöglichen), muss der verlinkte Text nicht mit irgendeinem der Terme im Verweisziel (Verweis-Konzept/-Frame) übereinstimmen. Beispielsweise kann die Angabe „medizinischen Produkten“ - vgl. Beispiel (3) - auf einen Frame verweisen, der die Terme medizinisches Produkt und Medizinprodukt (oder zumindest einen der beiden Terme) enthält.

Die Anlage der Verweise könnte noch komfortabler gestaltet werden, indem das System für die Filler Verweisziele vorschlägt, und zwar noch während bzw. kurz nachdem der Bearbeiter die Textfelder für diese Filler mit Inhalt füllt. Der Terminologe entscheidet dann, ob der entdeckte mögliche Zusammenhang korrekt ist und der Verweis angelegt werden soll. Umgekehrt könnte das System auch bei der Anlage neuer Konzepte und Terme herausfinden, wo in der Terminologiedatenbank dieses Konzept bereits erwähnt ist und dann die Verweise von Fillern bestehender Konzepten auf das neue Konzept vorschlagen.

\section{$5 \quad$ Fazit}

Im vorliegenden Beitrag haben wir den Versuch unternommen, den praktischen Nutzen von Frames am Beispiel des Aufbaus von Terminologiedatenbanken zu illustrieren. Leitend waren dabei insbesondere die Fragen, inwiefern konzeptuelle 
Informationen, die durch Frames erfasst werden, in der Terminologiearbeit genutzt werden können und wie Frames für die Terminologiearbeit fruchtbar gemacht und mithilfe marktüblicher Software modelliert werden können. Die erzielten Ergebnisse lassen sich in vier Punkten zusammenfassen:

1. Die in empirisch-linguistischen Untersuchungen gewonnenen Frames dienen dazu, komplexe Konzepte, Themen oder ganze Themenbereiche zu modellieren und in Terminologiedatenbanken für praktische Zwecke aufzubereiten. Richtschnur ist dabei weniger theoretische Konsistenz als praktische Relevanz. Bei der Datenkodierung im Rahmen eines Terminologiesystems können zwar Elemente der gewählten Repräsentationsform verlorengehen, jedoch ist es mithilfe eines Terminologiesystems möglich, wesentliche Bereiche der konzeptuellen Repräsentation abzudecken bzw. diese auf wesentliche Elemente zu beschränken. Auch können die Daten nach der Kodierung in unterschiedlicher Weise im Terminologiesystem gefiltert, visualisiert und im Standard-Austauschformat TBX weitergegeben werden.

2. Für die anvisierten terminologisch-lexikografischen Zielsetzungen erweist sich ein hybrides Frame-Konzept als brauchbar, das praktischen Zwecken flexibel Rechnung tragen kann. Es hat sich gezeigt, dass der in der Terminologieforschung vielfach verwendete Konzept-Begriff große Ähnlichkeiten zum FrameBegriff des KI-Forschers Minskys aufweist. Hilfreich ist insbesondere, dass Minsky - im Gegensatz zu Barsalous und Fillmores Ansatz - die Kategorie des Standardwertes („default assignment“) einführt (vgl. aber Ziem 2014a, 289 ff.). Diese erlaubt es, Datenkategorien (Slots) mit prototypischen, erwartbaren Fillern zu belegen, die durch abweichende Werte ,überschrieben ' werden können. Welche Slots zur Beschreibung einer Entität überhaupt in Frage kommen, bleibt indes offen; hierfür lässt sich die FrameNet-Datenbank nutzen, wie am Beispiel von Verpackungsmaschinen mithilfe des Placing-Frames demonstriert wurde.

3. Konkret können Frames für die praktische Terminologiearbeit in mehrfacher Hinsicht hilfreich sein. Frames helfen zum einen bei der Erfassung und Darstellung von Wissen. Viele Terminologiesysteme erlauben darüber hinaus bereits die Anlage von Templates und Verweisen. Sie lassen dem Terminologen auch große Freiheit bei der Anlage von Frames, Slots, Fillern und Verweisen. Dass logisch-formale Beziehungen nicht definiert werden müssen und nicht überprüft 
werden, ist von Vorteil, solange die Terminologiesammlung für menschliche Rezipienten konzipiert ist. Der Terminologe kann sich auf das konzeptuelle und sprachliche Wissen konzentrieren und es in einer Form darstellen, die für den aktuellen Kontext der Institution, ihrer Produkte und Prozesse am günstigsten ist. Die Anlage und Überprüfung logisch-formaler Beziehungen und Bedingungen z. B. in einem Wissensrepräsentationssystem oder einer formalen Ontologie wären ein zusätzlicher Zeitaufwand, den sich eine Institution angesichts begrenzter Ressourcen kaum leisten könnte. Ist der Rezipient hingegen ein Computerprogramm, das die Daten weiterverarbeitet, wäre zu hinterfragen, ob es sich überhaupt um eine Form von Terminologiearbeit handelt.

4. Terminologieverwaltungssysteme als Software könnten verändert oder erweitert werden, um neben der ,klassischen ' Terminologielehre auch alternative Ansätze besser zu unterstützen bzw. sich zunutze zu machen. Dies ist deswegen interessant, weil nicht alle Anwender das Ziel einer präskriptiven Terminologiearbeit verfolgen. Dabei kann eine Terminologiedatenbank entweder sowohl präskriptive als auch deskriptive Zwecke verfolgen oder mehr dem einen als dem anderen Zweck dienen. Ob einer der beiden Zwecke vorrangig erfüllt werden soll, bestimmen Faktoren wie konkreter Einsatzbereich, Unternehmensgröße, Unternehmenskultur, voraussichtliche Dauer der Tätigkeit in diesem Bereich usw. Durch die erlaubte Art der Datenmodellierung, die Prozesse zur Anlage der Terminologiebestände sowie durch die Möglichkeiten der Verwendung der erstellten Terminologiedatenbank sollte die Terminologieverwaltungs-Software das definierte Ziel bestmöglich unterstützen.

\section{Literatur}

Barsalou, Lawrence W. (1992): Frames, concepts, and conceptual fields. In: Kittay, Eva / Lehrer, Adrienne (eds.): Frames, fields, and contrasts: New essays in semantic and lexical organization. Hillsdale, NJ: Lawrence Erlbaum Associates, 21-74.

Busse, Dietrich (2012): Frame-Semantik. Ein Kompendium. Berlin: de Gruyter. Čulo, Oliver (2013): Constructions-and-Frames Analysis of Translations: The Interplay of Syntax and Semantics in Translations between English and German. In: Constructions and Frames 5 (2), 143-67. 
Das, Dipanjan / Chen, Desai / Schneider, Nathan / Smith, Noah A. (2013): FrameSemantic Parsing. In: Computational Linguistics 40 (1), 956.

Deutscher Terminologie-Tag e. V. (2014): Terminologiearbeit Best Practices 2.0. 2., überarbeitete und ergänzte Ausgabe. Köln.

Edition 2/2013: edition - die Terminologiefachzeitschrift. Ausgabe 2/2013: Terminologien und Ontologien. http://dttev.org/edition/63-terminologien-und-ontologien.html

Etzioni, Oren (2014): Knowledge Tour. Vortrag auf der Konferenz „The future of artifical intelligence and deep learning, San Francisco, 18. September 2014, http://new.livestream.com/gigaom/FutureofAI (letzter Zugriff: 1.12.2015)

Faber, Pamela / López Rodríguez, Clara Inés (2012): Terminology and specialized language. In: Faber, Pamela (ed.): A cognitive linguistics view of terminology and specialized language. Berlin / Boston: de Gruyter Mouton, 9-31.

Faber, Pamela (2012): A cognitive linguistics view of terminology and specialized language. Berlin, Boston: de Gruyter Mouton.

Faber, Pamela (2015): Frames as a framework for terminology. Handbook of Terminology, 1:14. John Benjamins Publishing Company.

Faber, Pamela / San Martín, A. (2012) Specialized language pragmatics. In: Faber, Pamela (Hrsg.): A cognitive linguistics view of terminology and specialized language. Berlin / Boston: de Gruyter Mouton, 177-203.

Fillmore, Charles / Baker, Collin (2010): A frames approach to semantic analysis. In: Heine, Bernd / Narrog, Heiko (eds.): The Oxford Handbook of Linguistic Analysis. Oxford: Oxford University Press, 313-340.

Fillmore, Charles J. (1968): The case for case. In: Bach, Emmon / Harms, Robert (eds.): Universals in Linguistic Theory. New York: Holt, Rinehart \& Winston, $1-88$.

Fillmore, Charles J. (1975): An alternative to checklist theories of meaning. In: Cogen, Cathy / Thompson, Henry / Thurgood, Graham / Whistler, Kenneth / Wright, James (eds.): Proceedings of the first annual meeting of the Berkeley Linguistics Society. Berkeley: Berkeley Linguistics Society, 123-131.

Fillmore, Charles J. (1985): Frames and the semantics of understanding. In: Quaderni di Semantica 6 (2), 222-254. 
Fillmore, Charles J. (2006) Frames Semantics. In: Brown, Keith (Hrsg.): Encyclopedia of Linguistics and Language. Bd. 4: Du - Gen. Amsterdam: Elsevier, $613-620$.

ISO 30042:2008. Systems to manage terminology, knowledge and content - TermBase eXchange (TBX). Geneva: ISO.

Lakoff, George (1987): Women, Fire, and Dangerous Things. What Categories Reveal about the Mind. Chicago: Chicago University Press.

Langacker, Ronald W. (1987): Foundations of Cognitive Grammar: Theoretical prerequisites. Stanford: Stanford University Press.

León Araúz, Pilar / Faber, Pamela / Montero Martínez, Silvia (2012): Specialized Language Semantics. In: Faber, Pamela (ed.): A Cognitive Linguistics View of Terminology and Specialized Language. Berlin / Boston: de Gruyter Mouton, 95-175.

Löbner, Sebastian (2014): Evidence from frames from natural language. In: Gamerschlag, Thomas / Gerland, Doris / Petersen, Wiebke / Osswald, Rainer (eds.): Frames and concept types. Applications to language and philosophy (= Studies in linguistics and philosophy 94). Heidelberg / New York: Springer, 23-68.

Lönneker, Birte (2003): Konzeptframes und Relationen. Extraktion, Annotation und Analyse französischer Corpora aus dem World Wide Web. Berlin: Akademische Verlagsgesellschaft AKA.

Lönneker-Rodman, Birte / Baker, Collin (2009): The FrameNet Model and its Applications. In: Natural Language Engineering 15 (3), 415-453.

López, Ana María Rojo (2002): Applying Frame Semantics to Translation: A practical Example. In: Translators' Journal 47 (3), 312-350.

Melby, Alan (2012): Terminology in the age of multilingual corpora. In: The Journal of Specialised Translation 18, 7-29.

Minsky, Marvin (1975): A Framework for Representing Knowledge. In: Winston, Patrick H. (ed.): The Psychology of Computer Vision. New York: McGrawHill, S. 211-277.

Minsky, Marvin (1986): The Society of Mind. New York: Simon and Schuster.

Petersen, Wiebke (2007): Representations of concepts as frames. In: The Baltic International Yearbook of Cognition, Logic, and Communication 2, 151-170.

Ruppenhofer, Josef / Ellsworth, Michael / Petruck, Miriam R. L. / Johnson, Christoph R. / Scheffczyk, Jan (2010): FrameNet II. Extended Theory and Practics. 
Berkeley: International Computer Science Institute. (abrufbar unter: https:// framenet2.icsi.berkeley.edu/docs/r1.5/book.pdf).

Rütten, Anja (2013): Terminologiesysteme im Dolmetscheinsatz. In: eDITion 1. Deutscher Terminologie-Tag e.V. (DTT), 25-28.

Sager, Juan C. (1990): A Practical Course in Terminology Processing. Amsterdam/Philadelphia: John Benjamins.

Ziem, Alexander (2013): Frames als Prädikations- und Medienrahmen: auf dem Weg zu einem integrativen Ansatz? In: Fraas, Claudia / Meier, Stefan / Pentzold, Christian (Hrsg.): Online-Diskurse. Theorien und Methoden transmedialer Online-Diskursforschung. Köln: Halem, 136-172.

Ziem, Alexander (2014a): Frames of Understanding in Text and Discourse: Theoretical Foundations and Descriptive Applications. (= Human Cognitive Processing 48). Amsterdam / Philadelphia: Benjamins.

Ziem, Alexander (2014b): Von der Kasusgrammatik zum FrameNet: Frames, Konstruktionen und die Idee eines Konstruktikons. In: Ziem, Alexander / Lasch, Alexander (Hrsg.): Grammatik als Inventar von Konstruktionen? Sprachwissen im Fokus in der Konstruktionsgrammatik. Berlin / New York: de Gruyter, 263-289. 